\title{
Blood utilization in elective surgery in a tertiary hospital in Dar es Salaam, Tanzania
}

\author{
LARRY O. AKOKO ${ }^{*_{1}}$ and ALEX B. JOSEPH ${ }^{2}$ \\ 'Department of Surgery, School of Medicine, Muhimbili University of Health and Allied Sciences, P.O. \\ Box 65,001, Dar es Salaam, Tanzania \\ ${ }^{2}$ Muhimbili National Hospital, Dar es Salaam, Tanzania
}

\begin{abstract}
Background: There has been an observation of blood being unnecessarily requested even for surgeries that will not require transfusion. Furthermore, some patients have been denied surgery due to lack of blood causing surgical list disruptions and inconveniency to patients and their families. This study was carried out to determine the status of blood utilization practices at Muhimbili National Hospital (MNH) in Dar es Salaam, Tanzania.

Methods: A descriptive cross-sectional study was carried out for a period of eight months. Data on blood requisition and usage was collected on a structured questionnaire, and analysed for Cross-match to Transfusion ( $\mathrm{C} / \mathrm{T})$ ratio, Transfusion probability (\% $)$, and Transfusion Index ( $\mathrm{TI})$. For those procedures that showed more than two indices to be significant, a Maximum Surgical Blood ordering Schedule MSBOS was proposed.

Results: Blood was over ordered for $76 \%$ of the patients while over eight percent of the patients had haemoglobin levels over $10 \mathrm{~g} / \mathrm{dl}$. Only amputation, laparotomy for malignancies, pneumonectomy, and bowel resection for malignancy had at least two of the transfusion indices showing significant blood utilization for general surgery cases. For urological cases, only Cystectomy for bladder cancer and nephrectomy had at least two of the indices showing significant blood utilization. In the remaining cases, blood utilization was inefficient suggesting cross-matching of blood was unnecessary, which resulted in loss of nearly $73 \%$ of technician's working hours and USD 425 for the study period.

Conclusion: There is existence of over ordering of blood in this hospital. Blood ordering practices needs to be reviewed to minimize over ordering of blood. All the three indices showed inefficient use of blood in most of the operations. But even so, utilization can still be improved in some of the operations which showed significant utilization.
\end{abstract}

Keywords: blood, transfusion, cross-matching, utilization, Tanzania

\section{Introduction}

Sub-Saharan Africa is still faced by inadequate supply of blood (WHO, 2006, 2011). It is reported that of the 92 million units of blood donated annually, only 4 miliion (4.3\%) are from the region which is a home to $12 \%$ of the global population. In order for Tanzania to meet its blood demand, it is recommended that at least 10 units per 1,000 populations should be collected annually (WHO, 2006). Tanzania National Blood Transfusion Services (NBTS) can only supply one third of the countries demands for safe blood and despite of the significant improvement in national blood collections (CDC, 2008), hospitals are still faced with serious blood shortages (Mbembati et al., 2008). The request of blood for elective surgical cases have been for a long time viewed as one of the areas of hospital wastage of this vital resource (Sowayan, 1994). Such requests are usually based on assumptions, thereby demanding large quantities of blood of which little is ultimately used. In order to save costs and technicians time, a number of studies have been conducted on blood ordering and transfusion practices in elective surgery (Ho \& Bo 2006;

\footnotetext{
*Correspondence E-mail: akokole12@gmail.com
} 
Friedman et al., 1976; Silberstein et al., 1989) and all demonstrated underutilization and overordering of blood.

Evaluating blood ordering and transfusion practices and subsequent developing of a blood ordering schedule, which serves as a guide to anticipated normal blood usage for elective surgeries can be the first step in decreasing over ordering of blood. This is the first study on requisition and transfusion practice in Tanzania. For this reason we carried out this study to determine efficiency of blood ordering and its subsequent utilization among elective adult urological and general surgical patients and to recommend a Maximum Surgical Blood Ordering Scheme for Muhimbili National Hospital.

\section{Materials and Methods}

\section{Study setting and data collection}

This study was carried out at Muhimbili National Hospital (MNH) in Dar es Salaam Tanzania. It was conducted between March and October 2013 involving all elective surgical patients in the Department of surgery. All consecutive patients who were operated on as elective cases and had blood requested and made available for perioperative use were recruited. Patient's particulars extracted from case notes included registration number, operative diagnosis, type of surgery performed, preoperative haemoglobin level, number of blood units cross-matched, number of blood units transfused intraoperatively and or within 72 hours post operatively were documented.

Muhimbili National Hospital has its own blood transfusion services, running independent from National Blood Transfusion Services and is fully dependent on replacement donation from relatives. Cross matching of blood in this hospital is done manually in the morning of the operation by one staff assigned hence hours worked in the shift were calculated and recorded. Therefore, we estimated from a mini survey that it takes one hour to cross match four units of blood. The cost of the reagent (Antihuman Globulin Serum-Coombs) was US\$83 for 80 units of blood hence money spent on unutilized blood was considered wasted. All the cross-matched blood of the day are then sent to the operating block in the morning where they are kept in ordinary refrigerators till the days surgical shift end in the evening. Thereafter unutilized blood would be sent back to the laboratory. However, blood bank inventory did not account for unused blood hence was considered discarded.

\section{Data analysis}

Data were cleansed, coded and entered into SPSS Version 16 and the following blood utilization indices. Cross-match to transfusion ratio ( $C / T$ ratio) was calculated as the number of units crossmatched per number of units transfused of which a value $<2.5$ indicates significant blood usage. Transfusion probability (\%T) was determined as the number of patients transfused per number of patients cross-matched multiplied by 100 (a value of 30\% indicates significant blood usage). Transfusion index $(\mathrm{TI})$ was calculated as the number of units transfused per number of patients cross-matched (a value of $>0.5$ is considered indicative of significant blood utilization). Maximal Surgical Blood Ordering Schedule (MSBOS) was calculated as 1.5 X TI (Friedman et al., 1976). This was calculated only for those procedures which had at least two of the indices showing significant blood utilization. The value denotes the units of blood to be cross matched.

\section{Ethical considerations}

This study received ethical approval from Muhimbili University of Health and Allied Sciences Institutional Review Board 


\section{Results}

During this study, blood was ordered for 445 patients undergoing elective urology and general surgical operations, of which only $23.6 \%$ (105/445) of them were transfused. There was no documentation of the status of the returned blood. The mean haemoglobin level of the patients' was $11.44 \pm 2.1 \mathrm{~g} / \mathrm{dl}$, with $82.5 \%$ (367) of the patients having value above $10 \mathrm{~g} / \mathrm{dl}$. Blood utilization was significantly increasing with decreasing haemoglobin levels ( $p<0.01)$. However, most of the blood was utilized by those patients with haemoglobin levels above $10 \mathrm{~g} / \mathrm{dl}$ (Table 1 ).

Table 1: Preoperative haemoglobin level and perioperative transfusion status

\begin{tabular}{llcll}
\hline Transfusion status & \multicolumn{2}{l}{ Haemoglobin concentration $(\mathrm{g} / \mathrm{dl})$} & \multicolumn{1}{l}{ Total } \\
Less than 7 & 7 to 10 & More than 10 & \\
Not transfused & $5(55.6)$ & $40(58)$ & $295(80.4)$ & $340(76.4)$ \\
Transfused & $4(44.4)$ & $29(42)$ & $72(19.6)$ & $105(23.6)$ \\
Total & $9(2)$ & $69(15.5)$ & $367(82.5)$ & $445(100)$ \\
\hline
\end{tabular}

A total of 565 units of blood were cross-matched of which only $27 \%$ (153) were transfused. Among 120 patients who underwent elective urology operations, 157 units of blood were cross matched of which $29.9 \%$ was transfused to $26 \%$ (31/120) of the patients. According to $C / T$, blood was appropriately ordered preoperatively for cystectomy+urinary diversion and nephrectomy only $(\mathrm{C} / \mathrm{T}<2.5)$. Transfusion probability was found to be high for the following operations: Cystectomy + urinary diversion, nephrectomy, open prostatectomy and ureteric exploration (\% T >30). On the other hand, transfusion index was found to be significant in cystectomy + urinary diversion and nephrectomy only ( $\mathrm{TI}>0.5)$ (Table 2$)$.

Table 2: Transfusion indices for urological operations $(n=120)$

\begin{tabular}{lllll}
\hline Type of operation & C/T ratio & $\begin{array}{l}\text { Transfusion } \\
\text { probability(\%) }\end{array}$ & $\begin{array}{l}\text { Transfusion } \\
\text { index (Ti) }\end{array}$ & MSBOS \\
\hline & & & & \\
Open prostatectomy (38) & $49 / 17,2.88$ & $13 / 38=34.21$ & $17 / 38=0.45$ & 0.67 \\
TURP (26) & $29 / 3,9.67$ & $3 / 26=11.54$ & $3 / 26=0.12$ & 0.18 \\
Urethroplasty (17) & $18 / 0, \infty$ & $0 / 17=0$ & $0 / 17=0$ & 0 \\
Nephrectomy (13) & $18 / 11,1.64$ & $7 / 13=53.84$ & $11 / 13=0.85$ & 1.27 \\
Ureteric exploration (6) & $9 / 2,4.50$ & $2 / 6=33.33$ & $2 / 6=0.33$ & 0.50 \\
Cystectomy +UD (6) & $16 / 13,1.23$ & $5 / 6=83.33$ & $13 / 6=2.17$ & 3.25 \\
Renal Stone extraction (6) & $8 / 0, \infty$ & $0 / 6=0$ & $0 / 6=0$ & 0 \\
Exploration laparotomy (renal & $4 / 1,4$ & $1 / 2=50$ & $1 / 2=0.5$ & 0.75 \\
malignancy) (2) & & & & \\
Bilateral Orchidectomy (1) & $1 / 0=\infty$ & $0 / 1=0$ & $0 / 1=0$ & 0 \\
Orchidopexy (1) & $1 / 0=\infty$ & $0 / 1=0$ & $0 / 1=0$ & 0 \\
Scrotoplasty (1) & $1 / 0=\infty$ & $0 / 1=0$ & $0 / 1=0$ & 0 \\
\hline Key: C/T=Cross-match to & & & \\
\hline
\end{tabular}

Key: $C / T=$ cross-match to transfusion ratio; $M S B O S=$ Maximum surgical blood ordering scheme/schedule

Among 325 patients who underwent elective general surgical operations, 408 units of blood were cross matched of which $26 \%$ (106/408) was transfused to $23 \%(74 / 325)$ of the patients. According to $\mathrm{C} / \mathrm{T}$ ratio, blood was appropriately ordered preoperatively for amputation, explorative laparotomy for malignancies, pneumonectomy and bowel resection for malignancies $(\mathrm{C} / \mathrm{T}<2.5)$. Transfusion probability was found to be high in the following operations: amputation, 
explorative laparotomy, pneumonectomy and bowel resection for malignancies (\% $\mathrm{T}>30$ ). Transfusion index was found to be significant in amputation, exploration laparotomy for malignancies, pneumonectomy, bowel resection, wide local excision, and cholecystectomy \pm CBD exploration ( $\mathrm{TI}>0.5$ ) (Table 3 ). 
Table 3: Transfusion indices for general surgery operations $(n=325)$

\begin{tabular}{|c|c|c|c|c|}
\hline Type of operation & $\mathrm{C} / \mathrm{T}$ ratio & $\begin{array}{l}\text { Transfusion } \\
\text { probability(\%T) }\end{array}$ & $\begin{array}{l}\text { Transfusion } \\
\text { index }(\mathrm{Ti})\end{array}$ & MSBOS \\
\hline Mastectomy (80) & $58 / 10=5.8$ & $10 / 80=12.5$ & $10 / 80=0.13$ & 0.19 \\
\hline Wide Local Excision (35) & $47 / 18=2.61$ & $10 / 35=28.57$ & $18 / 35=0.51$ & 0.77 \\
\hline Thyroidectomy (35) & $39 / 3=13$ & $3 / 35=8.57$ & $3 / 35=0.09$ & 0.13 \\
\hline Colostomy closure (34) & $41 / 7=5.85$ & $7 / 31=22.58$ & $7 / 41=0.17$ & 0.25 \\
\hline Amputation (31) & $45 / 22=2.05$ & $16 / 31=51.61$ & $22 / 31=0.71$ & 1.06 \\
\hline Bowel resection (Malignancy) (21) & $30 / 11=2.73$ & $8 / 21=38.09$ & $11 / 21=0.52$ & 0.79 \\
\hline Laparotomy (malignancy) (19) & $38 / 18=2.11$ & $11 / 19=58$ & $18 / 19=0.95$ & 1.42 \\
\hline CBD Bypass surgery (16) & $18 / 5=3.6$ & $4 / 16=25$ & $5 / 16=0.31$ & 0.47 \\
\hline Hernia repair (12) & $13 / 0=\infty$ & $0 / 12=0$ & $0 / 12=0$ & 0 \\
\hline Gastrectomy (6) & $8 / 2=4$ & $1 / 6=16.7$ & $2 / 6=0.33$ & 0.5 \\
\hline Haemorrhoidectomy (7) & $8 / 0=\infty$ & $0 / 7=0$ & $0 / 7=0$ & 0 \\
\hline Thoracotomy + pleural biopsy (5) & $7 / 2=3.5$ & $1 / 5=20$ & $2 / 5=0.4$ & 0.6 \\
\hline Cholecystectomy \pm CBD exploration (5) & $9 / 3=3$ & $1 / 5=20$ & $3 / 5=0.6$ & 0.9 \\
\hline Feeding Gastrectomy (5) & $5 / 0=\infty$ & $0 / 5=0$ & $0 / 5=0$ & 0 \\
\hline Peptic Ulcer surgery (3) & $5 / 0=\infty$ & $0 / 3=0$ & $0 / 3=0$ & 0 \\
\hline Microdoctomy (3) & $4 / 0=\infty$ & $0 / 3=0$ & $0 / 3=0$ & 0 \\
\hline Liver abscess drainage (3) & $4 / 0=\infty$ & $0 / 3=0$ & $0 / 3=0$ & 0 \\
\hline Pneumonectomy (2) & $7 / 5=1.4$ & $2 / 2=100$ & $5 / 2=2.5$ & 3.75 \\
\hline Gastric Polypectomy (1) & $1 / 0=\infty$ & $0 / 1=0$ & $0 / 1=0$ & 0 \\
\hline Heller's myotomy (1) & $2 / 0=\infty$ & $0 / 1=0$ & $0 / 1=0$ & 0 \\
\hline Anal Fistulectomy (1) & $1 / 0=\infty$ & $0 / 1=0$ & $0 / 1=0$ & 0 \\
\hline
\end{tabular}

Key: $C / T=$ cross-match to transfusion ratio; MSBOS=Maximum surgical blood ordering Scheme/schedule

During the study period (171 days), two technicians worked for 10 hours per shift for a total of 1,710 hours each. A total of 5,942 units of blood were cross matched during study period. On average 4 blood units were cross-matched per hour (5942/1710). Therefore, a total of 103 hours (412 unused units/ 4units) were lost on processing blood which was not utilized peri-operatively. Since a total of 656 units of blood were cross-matched for elective urological and general surgical procedures during study period, this means that a total of 141 hours (565/4) were spent to crossmatch these units of blood. This amounted to 73\% (103/141) of technician working hours being lost.

The cost of cross-matching one unit of blood was found to be about US\$1r, Since one $10 \mathrm{ml}$ bottle of reagent for cross-matching blood (Antihuman Globulin Serum-Coombs) costs US\$ 83 and would be used for up to 80 units, the cost of cross-matching 412 unused blood was US\$ 425 during study period.

\section{Discussion}

This study has provided insight into blood utilization in elective surgery at the only National Hospital in Tanzania. However, being a complex tertiary hospital, the results might not echo the practice in other hospitals. However, the findings are likely to reflect the practice in other hospitals since it plays the role model by virtue of being the oldest medical teaching institution in the country. Also advantaged by having its own blood transfusion services, Muhimbili National Hospital stands a better chance of improving its blood utilization from within changes rather than imposed from outside. This is the first study of this kind in the country and the region hence gives 
an opportunity to comment on efficiency of blood utilization and compare how the institution fares in comparison with others.

We have demonstrated from this study that there is existence of over ordering of blood in this hospital, with three of four cross-matched bloods being unutilized. Similar findings have been reported by other studies elsewhere (Abdelhadi, 2001; Basnet et al., 2009; Musa et al., 2014). The reasons for the over ordering in this study were not investigated. However, it can be speculated to be long traditions held by the surgical team and lack of trust that the laboratory will provide blood when the need arise during surgery. Improvement in blood ordering practices will save significant units of blood that might be utilized by other category of patients who need them on emergency basis. Even though this study did not look at the demand of blood by other category of patients, we still believe that some patients might have missed blood as it is not an infrequent phenomenon here.

The study revealed an overall C/T ratio of 3.7, inefficient utilization, being higher among general surgery operations compared with urology patients, findings that are similar to Egypt and Malaysian studies (Jayaranee, 2002; Ibrahim et al., 2011) but higher than in Nigeria and India (Bo et al., 2006; Subramanian et al., 2012). This study shows that C/T ratio varies widely across the surgical procedures from 9.67 (TURP) -1.23 (Cystectomy + Urinary diversion in Urological operations and 13 (Thyroidectomy) - 1.4 (Pneumonectomy) in general surgical operations. But using $\mathrm{C} / \mathrm{T}$ ratio alone might not be adequate, restrictive blood transfusion practice might improve some of the variables which showed significant utilization. For instance, transfusion in amputation can be completely avoided if tourniquets are appropriately applied and patients are properly prepared. It could not be established why blood was used in amputation.

The overall transfusion probability was also found to be low, similar to findings from India (Vibhute et al., 2000). This means that the probability of transfusions were inefficient in most of the procedures except for open prostatectomy, nephrectomy, ureteric exploration, cystectomy + urinary diversion, amputation, bowel resection, explorative laparotomy and pneumonectomy.

Overall transfusion index found in this study was 0.34 , reflecting inappropriate cross-matching of blood in most of the operations except for nephrectomy, cystectomy + urinary diversion, wide local excision, amputation, bowel resection, explorative laparotomy, cholecystectomy $\pm \mathrm{CBD}$ exploration, pneumonectomy. A study in India reported similar findings (Vibhunte et al., 2000).

The consequences of inefficient utilization of blood can also be viewed in monetary terms and work overload by staff cross-matching blood since it is performed manually. We have demonstrated that about three quarters of technicians working hours were wasted crossmatching bloods that were subsequently not utilized. This is much higher than in the findings of a similar study in Kuwait (Baraka et al., 1991). Similarly, the cost of cross-matching unused blood in this study was enormous. Yet, this was only for one department only. The cost would likely to be higher if other procedures such blood collection, processing and storage were considered. This means that the hospital might be losing substantial amount of money per year for all surgical departments including obstetrics and gynaecology, plastic surgery, paediatric surgery, open heart surgery, maxillofacial surgery and other surgical procedures that need blood transfusion.

From our findings, that would mean three quarters of the patients who were operated but not transfused would not have had their surgeries if blood was not made available. Postponement of surgical operation due to lack of blood at Muhimbili National Hospital has been reported by Mbembati et al., (2008). It is not easy to generalize that the precautionary ordering of blood by the surgical team at MNH is out dated, but from a joint discussion involving the blood bank that we carried out post study revealed a serious lack of trust that the blood bank will respond immediately when blood is needed in the operating room.

The findings of this study are likely to be useful for planning and establishing Maximum Surgical Blood Ordering Schemes at Muhimbili National Hospital. This will form the first step in 
reducing type of procedures that have blood cross-matched and made available for theatre but also on the number of units prepared per procedure. Such a move has been shown by Mujeeb et al. (2001) to be effective in improving blood utilization especially for elective surgical procedures. In conclusion, there is existence of over ordering of blood in this hospital. Blood ordering practices needs to be reviewed to minimize over ordering of blood. All the three indices showed inefficient use of blood in most of the operations. But even so, utilization can still be improved in some of the operations which showed significant utilization.

\section{Competing Interests}

The authors declare that they have no competing interest

\section{Authors' contributions}

Both authors contributed equally towards the accomplishment of this work and have all read and approved the final version of the manuscript.

\section{Acknowledgements}

This study was conducted with financial support from the Ministry of Health and Social Welfare and Muhimbili University of Health and Social Sciences. We highly acknowledge the support.

\section{References}

Abdelhadi, M., Layla, M.A. \& Bashawari, SA. (2001) Blood conservation in elective surgery. Kuwait Medical Journal 33, 232-234.

Baraka, A., Juma, T., Asfar, S.K. \& Al-Sayer, H. (1991) Conserving blood in preparation of elective surgery. A prospective study. Journal of the Royal Society of Medicine 84, 600-601.

Basnet, R.B., Lamichhane, D. \& Sharma, V.K. (2009) A study of blood requisition and transfusion practice in surgery at Bir Hospital. Postgraduate Medical Journal of NAMS 9, 14-19.

CDC (2008) Progress toward strengthening blood transfusion services---14 countries, 2003--2007. MMWR Weekly 57, 1273-1277.

Friedman, B.A., Oberman, H.A., Chadwick, A.R. \& Kingdon, K.I. (1976) The maximum surgical blood order schedule and surgical blood use in the United States. Transfusion 16, 380387.

Ho, O. \& Bo, B. (2006) Blood utilization in elective surgical procedures in Ilorin. Tropical Journal of Health Sciences 13, $15-17$.

Ibrahim, Z.S., Mamdouh, H.M. \& Ramadan, A.M. (2011) Blood utilization for elective surgeries at main University Hospital in Alexandria, Egypt. Journal of American Science 7, 683-689.

Jayaranee, S. (2002) An analysis of blood utilization for elective surgery in a tertiary medical centre in Malaysia. Malaysian Journal of Pathology 24, 59-66.

Mbembati, N.A., Mwangu, M., Muhondwa, E.P.Y. \& Leshabari, M.M. (2008) Performance Indicators for Quality in Surgical and Laboratory Services at Muhimbili National Hospital (MNH) In Tanzania. East African Journal of Public Heath 5, 13-16

Mujeeb, S.A. (2001) An audit of blood crossmatch ordering practices at the Aga Khan University Hospital: first step towards a maximum surgical blood ordering schedule. Journal of the Pakistan Medical Association 51, 379-380. 
Musa, A.U., Ndakotsu, M.A., Hassan, A.A., Kilishi, A. \& Kwaifa, I.K. (2014) Pattern of blood transfusion request and utilization at a Nigerian University Teaching Hospital. Sahel Medical Journal 17, 19-22.

Silberstein, L.E., Kruskall, M.S., Stehling, L.C., Johnston, M.F., Rutman, R.C., Samia, C.T., Ramsey, G., Eisenstaedt, R.S. (1989) Strategies for the review of transfusion practices. Journal of the American Medical Association 262, 1993-1997.

Sowayan, S.A. (1994) Use of blood in elective surgery: an area of wasted hospital resource. Annals of Saudi Medicine 14, 326-328.

Subramanian, A., Sagar, S., Kumar, S., Agrawal, D., Albert, V. \& Misra, M.C. (2012) Maximum surgical blood ordering schedule in a tertiary trauma center in northern India. Journal of Emergencies, Trauma and Shock 5, 321-327.

Vibhute, M., Kamath, S.K. \& Shetty, A. (2000) Blood utilization in elective general surgery cases: requirements, ordering and transfusion practices. Journal of Postgraduate Medicine 46, 13

WHO (2011) Blood safety key global facts and figures in 2011. Geneva, Switzerland: Available at http://www.who.int/worldblooddonorday/media/who_blood_safety_factsheet_2011.pdf. Accessed November 15, 2011.

WHO (2006) Status of blood safety in the WHO African Region: report of the 2006 survey. Brazzaville, Republic of Congo. Available at http://www.afro.who.int/en/divisions-aprogrammes/dsd/health-technologies-a-laboratories.html楁. Accessed November 15, 2011

WHO (2009) Blood safety and availability: facts and figures from the 2007 Blood Safety Survey. Geneva, Switzerland. Available at.

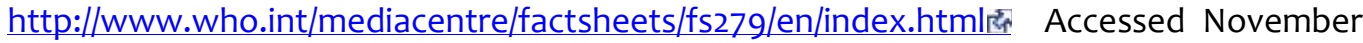
15, 2011. 